\title{
A NEW VIDEO CODING APPROACH TO THE FUTURE WIRELESS COMMUNICATION SYSTEM
}

\author{
Sarmad K. Ibrahim ${ }^{1}$, Nasser N. Khamiss ${ }^{2}$ \\ ${ }^{1}$ College of Engineering,Al-Mustansiriyah University,Baghdad,Iraq, \\ ${ }^{2}$ College of Information Engineering,Al-Nahrain University,Baghdad,Iraq \\ eng-sarmadnet@uomustansiriyah.edu.iq ${ }^{1}$,nasser@coie-nahrain.edu.iq ${ }^{2}$ \\ Received:30/4/2019, Accepted:3/6/2019
}

\begin{abstract}
The current environment of video systems is heterogeneous due to the variety of the devices used in the display such as HD and diversity of data transfer rate from one client to another, which leads to diversity in the resolution of the video for each client. The heterogeneous network systems increase the storage of video files, although that the $\mathbf{H . 2 6 5}$ reduces the rate of data about to half as compared to $\mathbf{H . 2 6 4}$, but it stores as the multiple video resolutions of one video on the system in the heterogeneous system to satisfy different consumer characteristics and is delivered exploitation broadcast coding. $\mathrm{H} .265$ is used as a multi-layer encoding to provide a different video resolution, which is increased the complexity of encoding system and time. The paper solves such problems by proposing a new technology for encoding,which depends on scalable core video technique in order to save a bit rate and improve the quality. The complexity of the encoding system and time is reduced by prediction the upper layers of video resolution from the lower layer in new technology. The results present that the proposed technique is better than H.265 transcoding, the rate of data transfer which has reduced (about $47 \%$ ) in $\mathrm{HD}$ as compared to the H.265, while the PSNR has increased (about 3dB) in the same type of resolution. Furthermore, the processing time is decreased to $14 \%$ as compared to H.265.The complexity is reduced to $25 \%$ as compared to $\mathrm{H} .265$.
\end{abstract}

keywords: transcoding, Interlayer reference, sublayer, reference prediction.

\section{INTRODUCTION}

Last years, the service of video consumption in high resolution was increased (such as UHD and 8K), which it distributes over the broadcast channels, computer networks, or storage media. Such associate rising trend creates a much bigger demand on the event of video acquisition, compression, and show technologies, also as of network and transmission media capabilities. The foremost crucial requirements arise during a video compression domain, where it's necessary to satisfy the need for a very economical video coding commonplace [1]. H.265 was estimated to decrease the rate of the bit compared to Advanced Video Coding (H.264) while maintaining similar quality[2].

A fresh video compression which is more flexible and like an HEVC, the High Definition handy client's devices may require additional HD evolution in traffic of mobile video[3]. Several tablets already have resolutions of 8k probable in the 2020s .H.265 established by professionals from ISO/IEC and ITU- T, standardization activity was begun from 2010 and available the essential version of coding standard in 2013 [4].

Recently, video service over IP networks has been increased, video services presently are approximate $73 \%$ of all the traffic services are consumed across networks and are expected to be consumed $82 \%$ in 2021[5]. Also, Mobile devices increasing the bit rate to twice of IP fixed[6]. Also, as 5G mobile systems enter service[7], estimated higher bandwidths, improved reliability and lower delays, are possible to arise the video on demand over the mobile system. These days, the drift is to provide expanded quality of video administrations to clients persistently. For higher quality, it is essential to utilize high-performing encoders since the transmission capacity of the transmission line is constrained. Later codecs are more difficult and need extra processing time to encode video. It is unsatisfactory to spend a few hours compressing 
a brief video arrangement in High Definition (HD) [8]. The improved of H.265 has many previous works. In [9], the authors compared to H.265 lossy coding and lossless coding and proposed a new sample-based angular intra prediction (SAP) method to enhance H.265 lossless mode coding efficiency further. Authors studied the coding of intra in H.264 and H.265 with common coding of the image such as JPEG 2000 and JPEG to present the bit-rate for H.264 and H.265 intra coding. In[10], the authors evaluated and compared between VP9, H.264, and H.265[11]. Authors[10] analyzed the H.265 using the common encoder tool (HM) rate distortion and complexity and evaluated the results of all - intra (AI) AVC reference encoder, low delay B, low delay $\mathrm{P}$, and random access configurations [12]. Authors compared between H.265 and H.264 and analyzed throughput using the Bjontegaard delta measurement in paper [13]. In [14], the authors proposed an approach to employ the encoding data to limit the rate-distortion - optimization of the encoding, the encoding complexity was reduced while maintaining high rate-distortion performance. Recently, authors evaluated the two modern video coding standards H.264 and H.265 over LTE advanced system, to find the best video codec method[15]. The goal of the paper proposes a video transcoding method that is called Pro technique. It is compared with H.265 coding to find the best performance in terms of bit rate and PSNR (overhead).

\section{Advantages of the proposed method as compared to the H.265 transcoding method}

- Low bit rate $(20-46 \%)$.

- High PSNR (1-3 dB).

- Low complexity $(25 \%)$.

- Low processing time $(14 \%)$.

\section{OVERVIEW OF H.265}

H.265 procedures outlined the configurations to denote the coded data of video, as shown in Fig. 1. H.265 provides blocks, which are taken in Network Abstraction Layer[16]. H.265 has many improvements such as partitions flexibility and more interpolation that is a complicated and unblocking filter, a prediction that is new complicated and motion vector (MV), and support a multi-process [4], [16], [17]. H.265 has a different block coding structure, which are:coding tree units (CTU)and coding tree block(CTB), prediction units(PU) prediction blocks(PB), transform units(TU), transform blocks(TB)[18]-[21]. Recently, there is a wide range of systems that receive digital video data such as, tablets, TVs, mobile phones and PC; each has different computational, display, and connectivity capabilities, this video has to be converted to meet the specifications of the target system. This conversion is achieved through video transcoding. For transcoding, a straightforward solution is to decode the compressed video signal and re-encode it to the target compression format, but this process is computationally complex, as shown in Fig. 2. The complexity is solved by the proposed video coding in the next section.

\section{Proposed Video Coding Technique (Pro Technique)}

The video in the improved video coding method is coded in several layers, in terms of spatial resolution, each layer presents a version of the same data of video. A method has layer zero which is called a base (BL), and enhancement layer (EL) layer of, EL is coded on the BL which is contained the enhanced video, as shown in Fig. 3, it is useful for connection having variable link rate (such as $4 \mathrm{G}$ ). It is applied for different application such as, surveillance, which is 


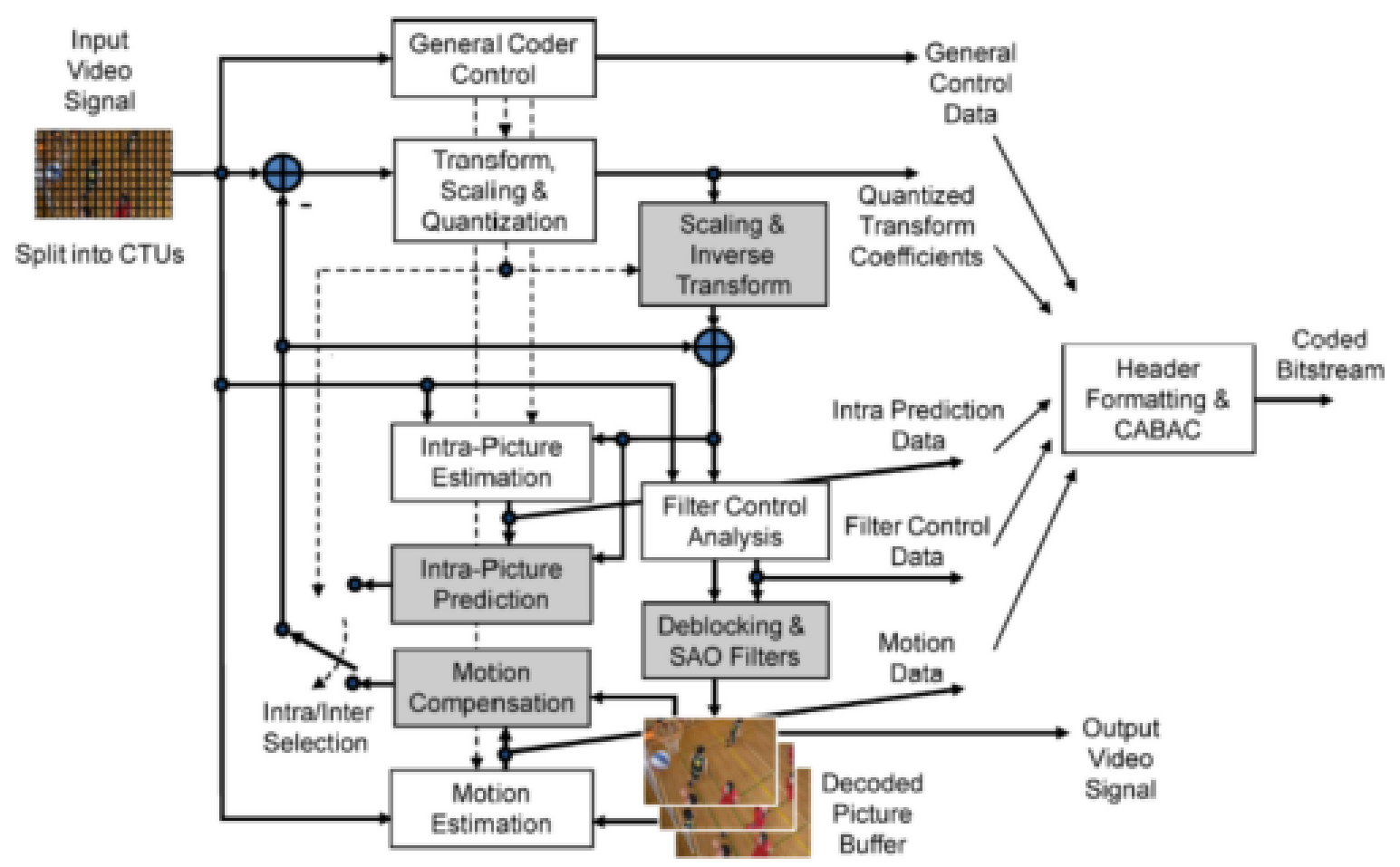

Figure 1: The encoder of H.265[15]

not viewed on multiple devices of the monitor but wants to store and archive. It has numerous advantages such as less rate of data, more resilience to layer combination. A method has several features such as less data rate in the broadcast application, less storage space, additional flexibility, and supports up and downsampling. Pro technique method philosophy permits scalability execution using double of a single layer in the H.265 to obtain high efficiency of coding. Pro technique reprocesses all the compression stages of $\mathrm{H} .265$ beside prediction of the interlayer, which finds the redundant data between two layers to achieve an efficiency of coding by forecasting the EL layer using the BL. The temporal scalability of Pro technique refers to temporary sublayers. The operating point defines subsets of temporary sublayers, which able to decode. The temporary has identified the prediction of temporal constraints which is called ID value between the frames, and a frame with a higher temporary ID cannot be used as a reference at a particular temporary ID value. An access unit contains one, or a lot of coded photos; the layer info is indicated in the NAL unit header and video parameter set (VPS). The NAL contains data concerning the temporal sub-layer and layer. The VPS includes data of the layer (types, dependencies and sets, operation points, output sets, and layer hypothetic reference decoder info). The syntax style of Pro technique is changed such that the set of restored frames (resampled if necessary) from the BL by using the inter-layer reference picture (ILR) once coding the present EL frame, which permits prediction of inter-layer to apply with none low-level coding method changes as shown in Fig. 4. ILR frames are created by three main modules: resampling textures and resampling motion 


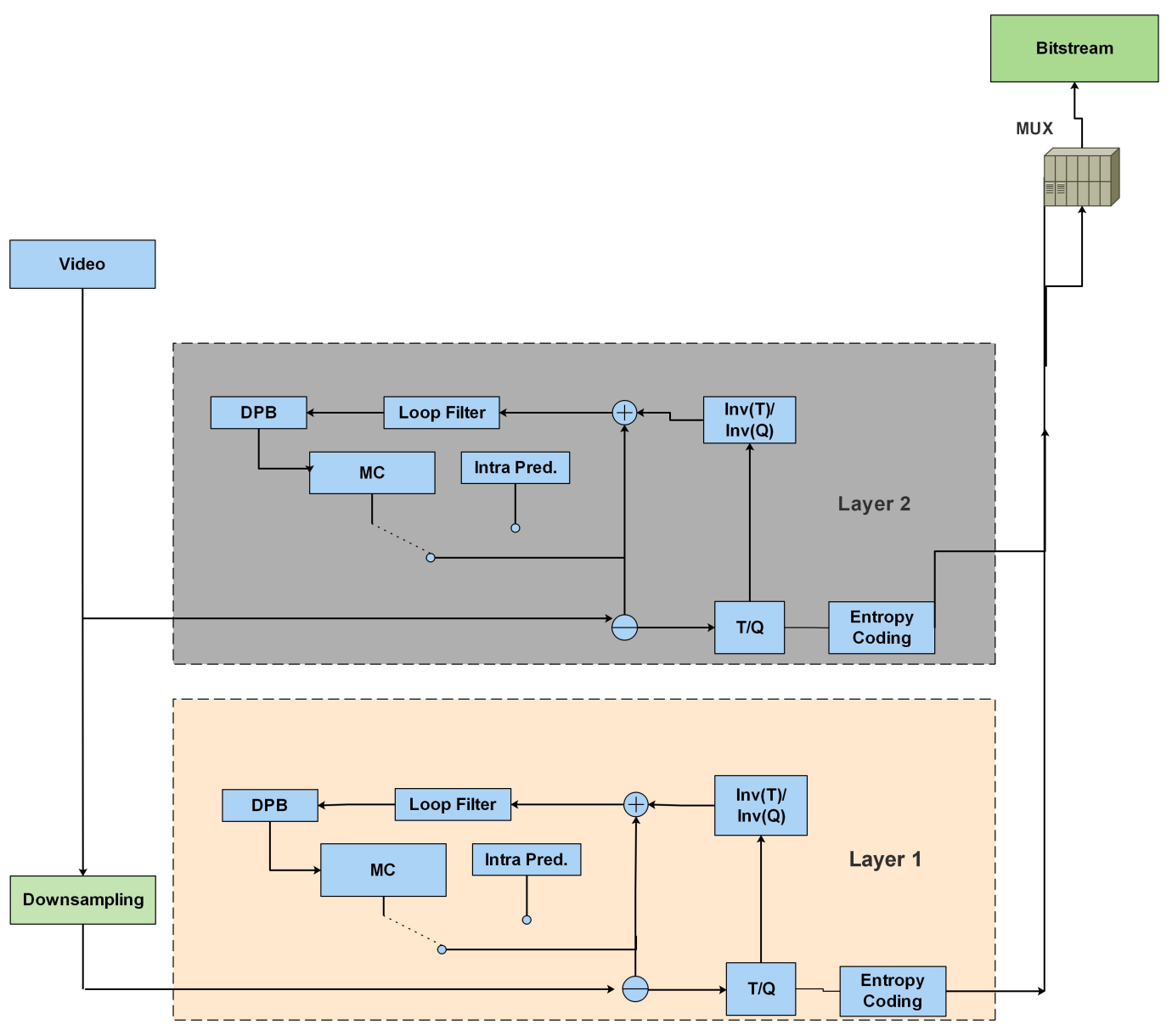

Figure 2: Sample layering and sub-layering in H.265(standard) bit-stream

field for spatial scalability and bit depth. The lists of the initial reference pictures in Pro technique are created as follows: in the picture list 0 (L0), the ILR pictures are added below the temporal reference pictures for the time reference pictures and the set of temporary reference pictures for the temporary reference pictures are inserted in reverse. the pictures list 1 (L1) is created by the temporal references are first inserted into the list of references in a similar way as the structure of the list of initial pictures in H.265. Furthermore, the ILR image (s) is added as a long-term reference image (e) at the end of L1. The current EL image is coded as P-slice from the list of reference images L0 when the current EL image is coded as B-slice from reference images L0 and L1. The up-sampling filters are a set of filters with an accuracy of 1/16. Pro technique uses a finite impulse response (FIR) polyphase filter of 8 taps for resampling luma and a polyphase FIR filter with 4 taps for chroma scanning. The upstream scan filters are designed with the interpolation filter of motion, which has 0.25 accuracy for luma and 0.125 chroma precision in the initial model of H.265. 


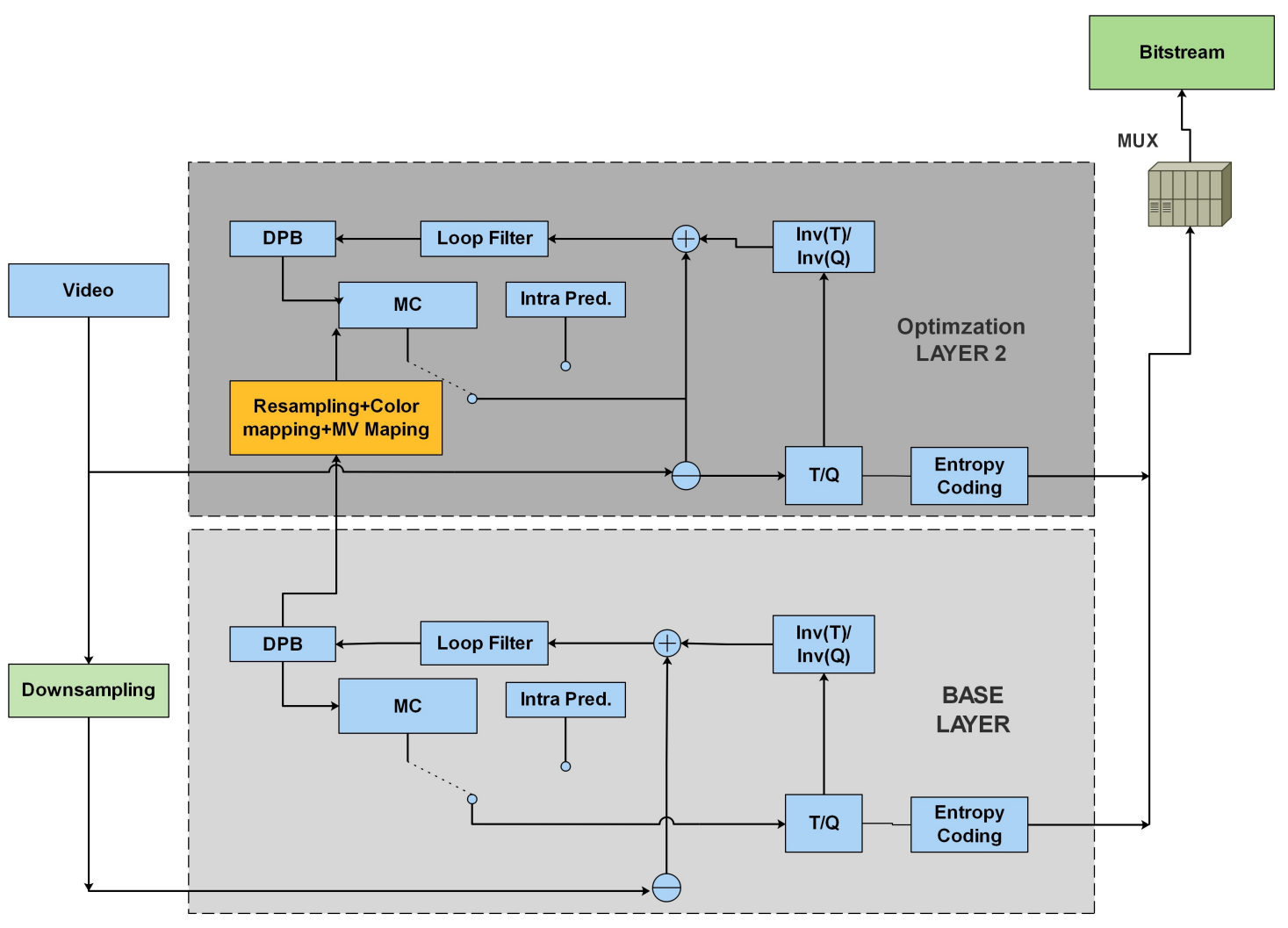

Figure 3: Sample Layering and Sub-layering in pro technique bit-stream

\section{Simulation AND Results}

An FFMPEG, HM 16 tools have been used to apply the Pro technique on the raw video data (HD). The pro technique is presented in Fig. 5. The raw video (HD) is applied the downsampling into three layers (720 HD, 4CIF, CIF). The raw video data as presented in Table I. In the First stage of Pro technique, the base layer (CIF) is encoded the video data, then, the enhancement layer 1 (4CIF) is predicted the information from the base layer (DPB) after resampling of the video data, then, the enhancement layer $2(4 \mathrm{CIF})$ is also predicted from the enhancement layer 1 if the data rate of wireless channel is high. The process is continued until the level of the raw video is reached. Finally, the encoded data in layers are mixed and transmitted and is transmitted via the wireless channel. Finally, the decoded data is received by the receiver. Fig. 6 presents the sequence encoding layers in video encoding tool. 


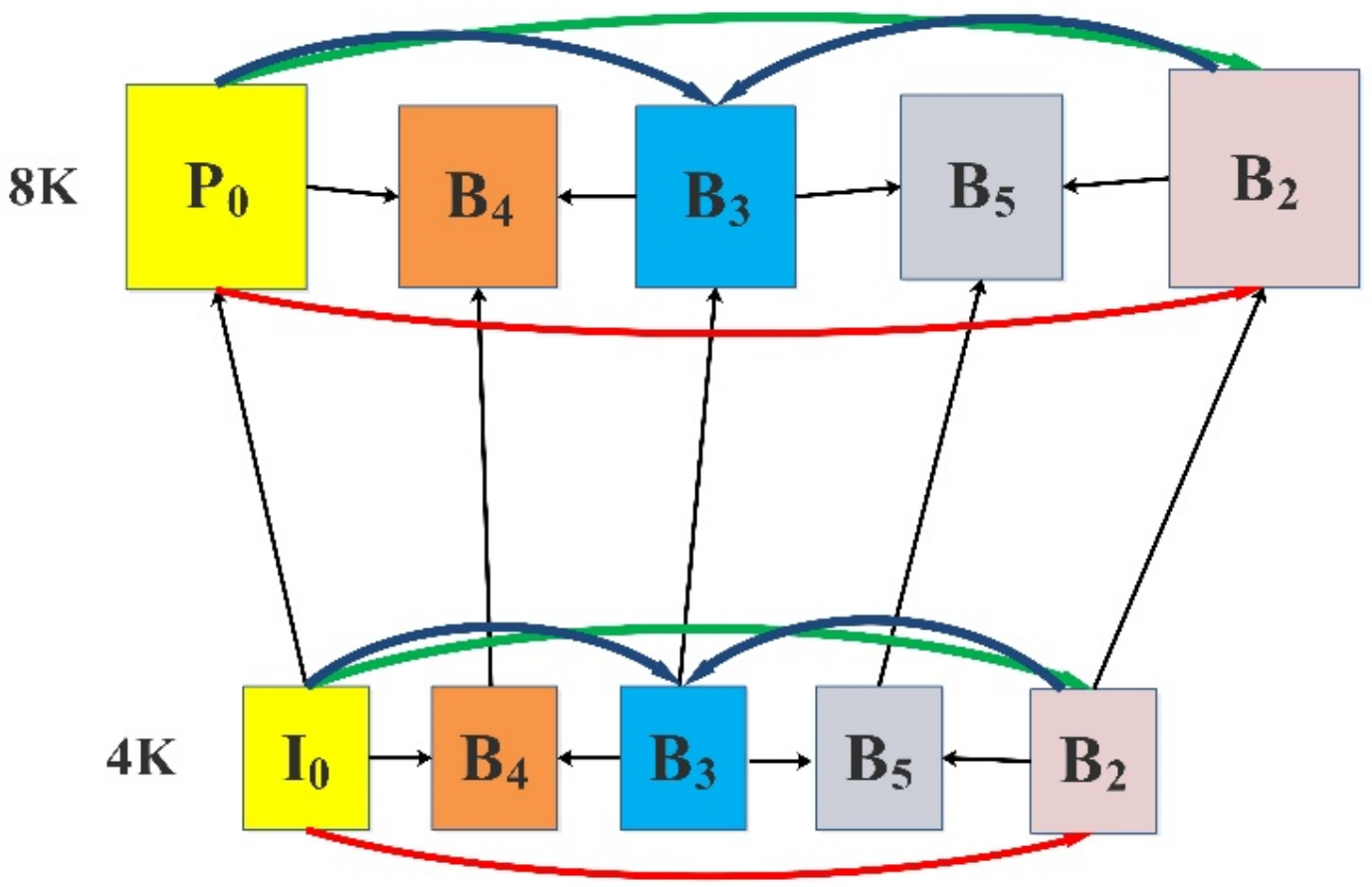

Figure 4: Inter-layer prediction

TABLE I

RESOLUTION OF THE RAW VIDEO

\begin{tabular}{|c|c|c|c|}
\hline Name of Resolution & Resolution & Frame rate & Number of Frames \\
\hline CIF & $352 * 240$ & & \\
\cline { 1 - 2 } 4CIF & $704 * 480$ & \multirow{2}{*}{20} & \multirow{2}{*}{120} \\
\hline 720 HD & $1280 * 720$ & & \\
\hline HD & $1920 * 1080$ & & \\
\hline
\end{tabular}

Fig. 7 presents a bit rate at 480 video resolution between h265 and Pro technique of the video coding, the bit rate in both coding increases when the PSNR has been increased. The bit rate of Pro technique is lower than H.265 when the PSNR is $38 \mathrm{~dB}$, the bit rate in the Pro technique is around $678 \mathrm{kbps}$ on this as opposed to H.265 is around $774 \mathrm{kbps}$. In terms of overhead (PSNR), the Pro technique is higher PSNR than h.265 at $800 \mathrm{Kbps}$ about $1 \mathrm{~dB}$.

In 720 resolution, the Pro technique bit rate is better than $\mathrm{H} .265$ (about $658 \mathrm{kbps}$ and $911 \mathrm{kbps}$ respectively) at $38 \mathrm{~dB}$, the difference in PSNR between two coding is about 2dB, as presented in Fig. 8. When the resolution is 1080, the Pro technique bit rate is better than H.265 (about $525 \mathrm{kbps}$ and $988 \mathrm{kbps}$ respectively) at $40 \mathrm{~dB}$, the difference in PSNR between two video coding is about $2.5 \mathrm{~dB}$, as presented in Fig. 9. The Processing time in a Pro technique bit rate is better than H.265 (about $11877 \mathrm{sec}$ and $13844 \mathrm{sec}$ respectively) as presented in Fig. 10. Pro technique is used almost the coding data from the base layer to predict frames in the upper layers. Table II illustrates the result of a compression ratio. 


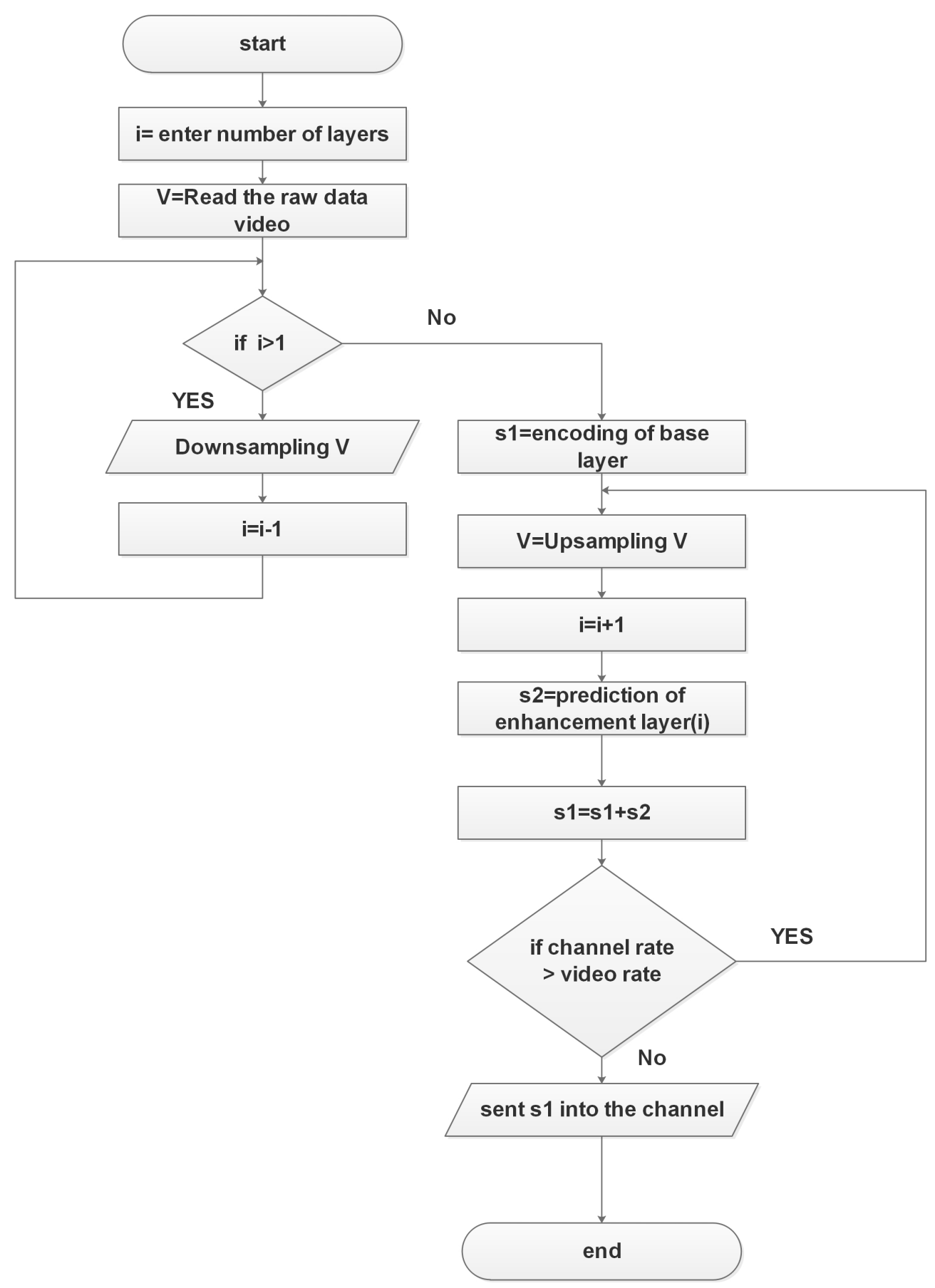

Figure 5: Simulation of Pro technique encoder 


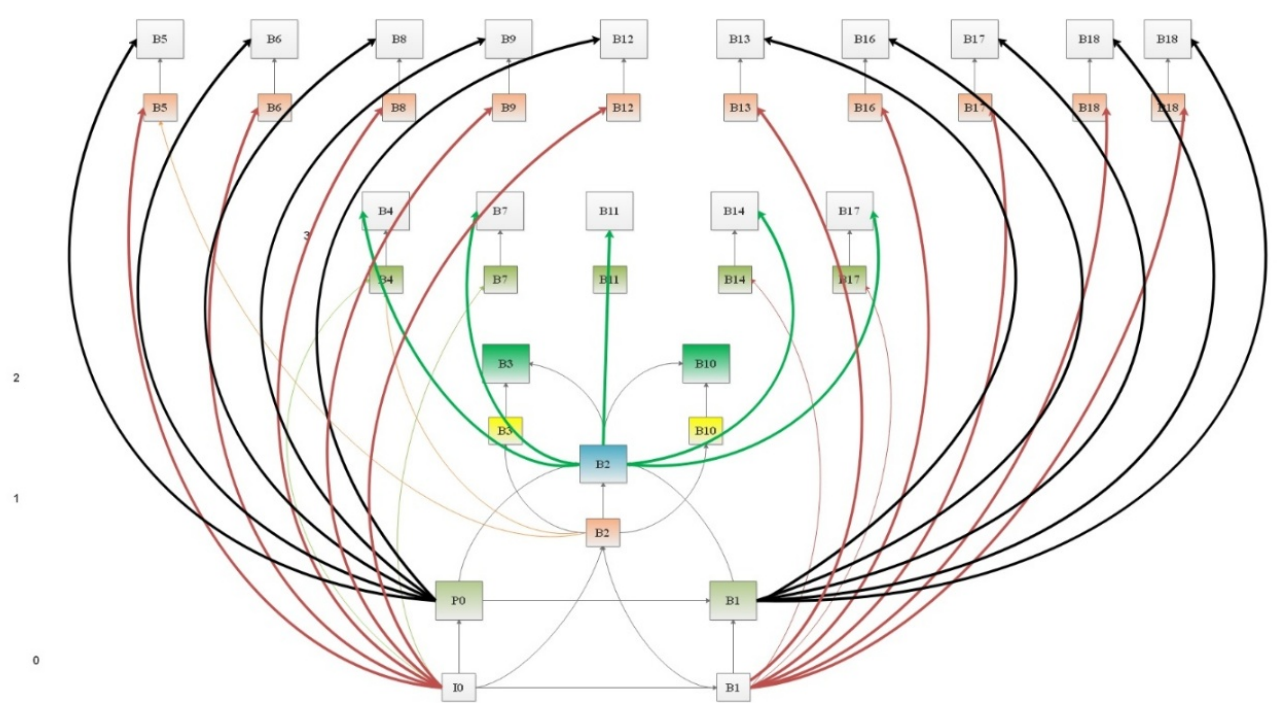

Figure 6: Simulation of frame decoding structure in Pro technique encoder

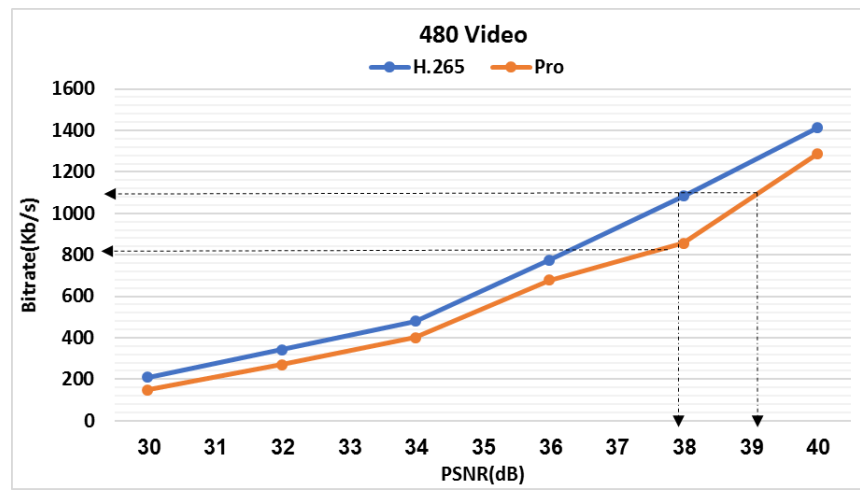

Figure 7: Comparison of The Bit rate Between the H.265 and The Pro Technique at 480 Video Resolution

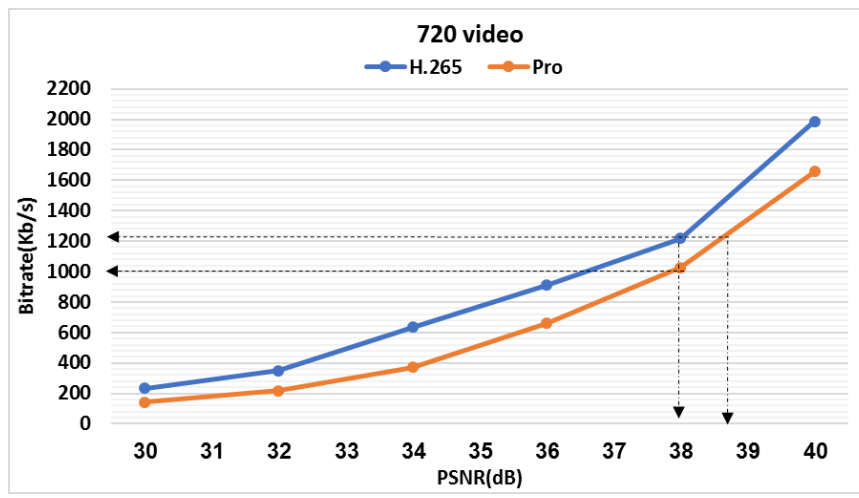

Figure 8: Comparison of the bit rate between the H.265 and the Pro technique at 720 video resolution 


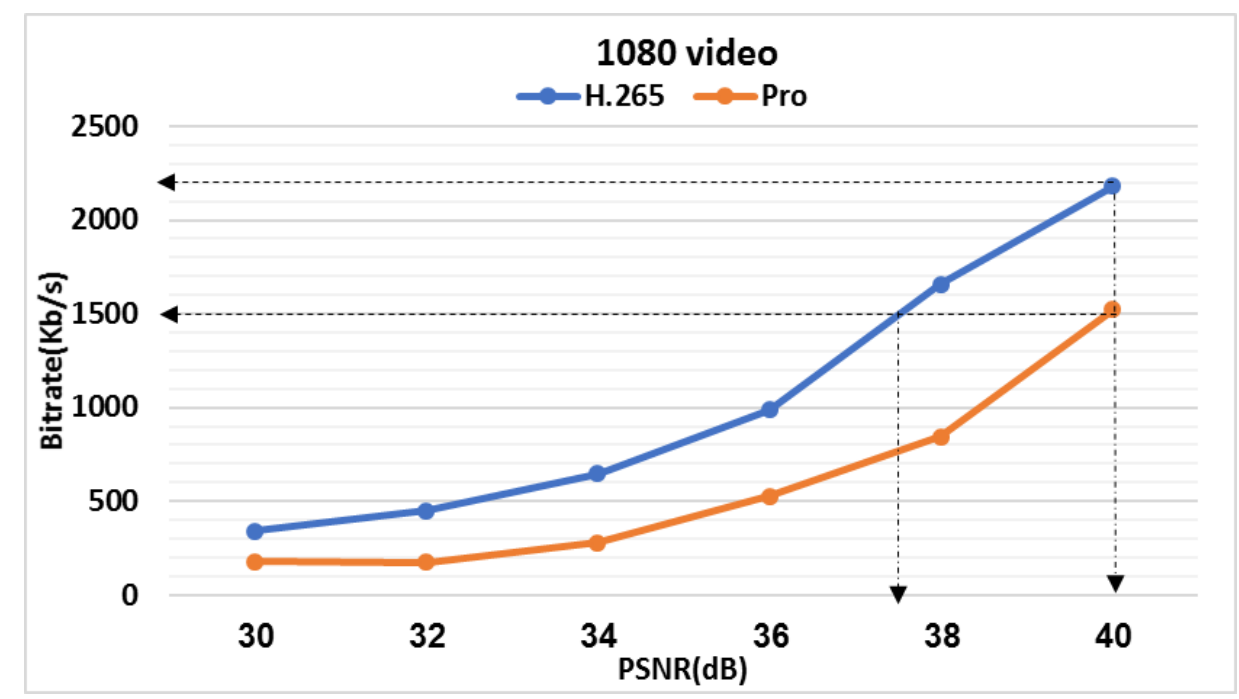

Figure 9: Comparison of the bit rate between the H.265 and the Pro technique at 1080 video resolution

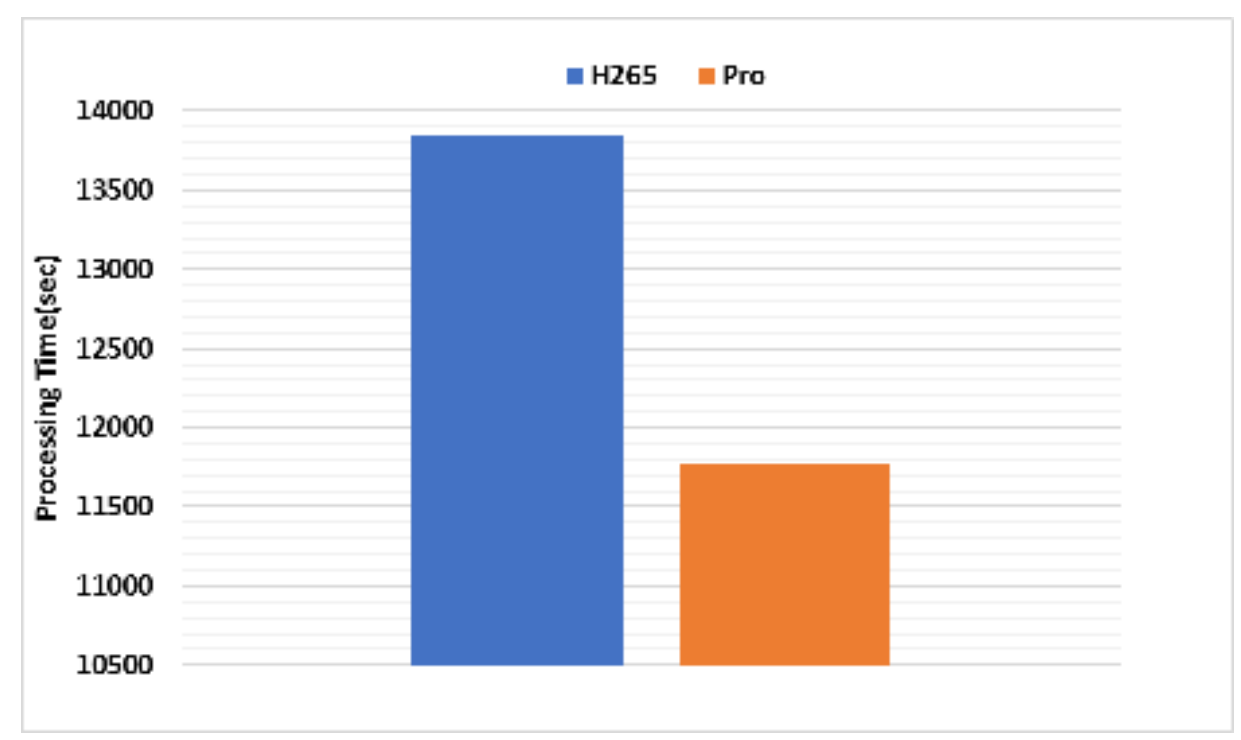

Figure 10: Comparison of the total processing time between the H.265 and the pro technique 


\begin{tabular}{|c|c|c|}
\hline \multirow{2}{*}{ Name of Resolution } & \multicolumn{2}{|c|}{ Compression ratio } \\
\cline { 2 - 3 } & Pro technique & H.265 \\
\hline 4CIF & 718 & 628 \\
\hline 720HD & 2015 & 1455 \\
\hline HD & 5682 & 3020 \\
\hline
\end{tabular}

The best performance in the Pro technique is shown in the result figures, which is exploited almost the redundant frames or pixel in the base layer and isn't exploited the redundant frames in enchantment layers. This feature is reduced the bit rate, the complexity, and the encoding time. The base layer only is encoded the video data; this is meaning that the higher layer is predicted from the base layer while the H.265 is encoded separately of each layer of resolution.

\section{Conclusions}

The paper proposes a method of video encoding, which is applied to reduce the bit rate, time processing, and storage by prediction upper layer, which is used in the future wireless communication such as $5 \mathrm{G}$ to provide the video service to support a lot number of customers. Pro technique has been compared with the traditional method (H.265) to find the best performance in terms of bit rate, PSNR, the compression ratio. The result has been showed the Pro technique outperforms H.265 in terms of the bit rate about to $47 \%$ in some of the video resolution cases, while the PSNR is increased in Pro technique about $3 \mathrm{~dB}$ as compared to H.265 at the same of bit rate. The best performance back to the Pro technique, which exploits the redundant frames or pixel in the base layer and not the need to discover them in another layer and also the base layer only encodes the video, and this is meaning which the higher layer is predicted from the lower layer(base) to reduce bit rate while H.265 is encoded separately of each layer of resolution, which is led to increasing complexity and encoding time while the complexity is decreased in Pro technique about 25\% as compared to H.265, time of encoding is also decreased about $14 \%$ compared to H.265.

\section{REFERENCES}

[1] M. T. E. Reabek, "Comparison of compression efficiency between HEVC/H.265 and VP9 based on subjective assessments", in SPIE,2014, pp. 1- 13.

[2] M. Wien, " High-Efficiency Video Coding - Coding Tools, and Speci cations", Berlin, Springer, 2014.

[3] J. Ohm, G. J. Sullivan, H. Schwarz ,T. K. Tan, S .Member, and T. Wiegand, "Comparison of the Coding Efficiency of Video Coding Standards - Including High-Efficiency Video Coding (HEVC)", in IEEE TRANSACTIONS ON CIRCUITS AND SYSTEMS FOR VIDEO TECHNOLOGY, 2012, vol. 22 ,no. 12 ,pp. 1669- 1684.

[4] G. J. Sullivan, J. R. Ohm, W. J. Han, and T. Wiegand, "Overview of the high- efficiency video coding(HEVC)standard", IEEE Trans. Circuits Syst. Video Technol. , vol.22,no. 12,pp. 1649- 1668, 2012.

[5] P. Hanhart, P. Korshunov, T. Ebrahimi, and M. Re, "Quality Evaluation of HEVC and VP9 Video Compression in Real- Time Applications," no. May 2015.

[6] H. Y.Sepehr Rezvani, Saeedeh Parsaeefard, Nader Mokari, Mohammad R. Javan, "Delivery-Aware Cooperative Joint Multi- Bitrate Video Caching and Transcoding in 5G", in Mobile Computing (TMC) ,2018, pp .1- 47.

[7] J. Nightingale, P.Salva- Garcia, J. M. A. Calero, and Q.Wang, "5G- QoE: QoE Modelling for Ultra-HD Video Streaming in 5G Networks", in IEEE Transactions on Broadcasting, 2018, pp. 1- 14.

[8] J. Kufa and T. Kratochvil, "Software and hardware HEVC encoding", in International Conference on Systems, Signals, and Image Processing, 2017, pp. $1-5$.

[9] M. Zhou, W. Gao, M. Jiang, and H. Yu, "HEVC lossless coding and improvements", EEE Trans. Circuits Syst .Video Technol. , pp. 1839- 1843, 2012.

[10] T. Nguyen and D. Marpe, "Performance analysis of HEVC- based intra coding for still image compression", Pict. Coding Symp. , pp.233- 236, 2012.

[11] D. Grois and A. Mulayoff, "Performance Comparison of H .265/ MPEG- HEVC, VP9,and", Pict. Coding Symp. IEEE, pp. $394-397,2013$. 
[12] T. D. H. J. Vanne, M. Viitanen, "Comparative rate- distortion-complexity analysis of HEVC and AVC video codecs", IEEE Trans. Circuits Syst. Video Technol. , pp. 1885- 1898, 2012.

[13] V. Sze and M. Budagavi, "A comparison of CABAC throughput for HEVC/H.265 VS.AVC/H.264", Signal Process. Syst. ,pp. 165- 170, 2013.

[14] D. Schroeder, A. Ilangovan, and M .Reisslein, "Efficient multi-rate video encoding for HEVC- based adaptive HTTP streaming", CIRCUITS Syst. VIDEO, Technol. , pp. 1- 15, 2016

[15] S. K. Ibrahim and N. N. Khamiss ,"Optimal Usage of LTE Advanced System to Support Multi- user in Video Streaming Application", 2018 Third Sci. Conf. Electr. Eng., pp. 197- 202, 2019.

[16] A. Jassal, I. S.Member, C.Leung, and I. Member, "H.265 Video Capacity over beyond-4G networks", Commun. QoS, Reliab. Model. Symp. IEEE ICC, 2016, pp. 1- 6, 2016.

[17] M. Winken, P. Helle, and D. Marpe, "TRANSFORM CODING IN THE HEVC TEST MODEL", IEEE Int. Conf. Image Process. , pp. 3693- 3696, 2011.

[18] K.Sihag and C.S.Lamba,"Algorithm and Architecture Design of High- Efficiency Video Coding( HEVC )Standard", Int. J. Comput. Sci. Mob .Comput. , vol. 5, no. 10, pp. 171- 178, 2016.

[19] R.Ranjana and D.K.Mahesh,"Video Compression Using Compact Tool( HEVC )", Int. J. Adv. Res. Comput. Sci. Softw. Eng. , vol. 6, no.7, pp. 140- 143, 2016.

[20] M. Uhrina, J.Frnda, L.Sevcik,and M.Vaculik, "Impact of H . 264/AVC and H .265/HEVC Compression Standards on the Video Quality for 4K resolution", Adv. Electr. Electron. Eng. J, no .December, pp. 368- 376, 2014.

[21] S.K.Ibrahim and N. N. Khamiss, "A New Wireless Generation Technology for Video Streaming", J. Comput. Networks Commun., vol. 2019, pp.19, 2019. 